INRA Prod. Anim.,

2014, 27 (5), 359-368

\title{
Aménagement des logettes et confort des vaches laitières
}

\author{
P. CAZIN, B. NICKS, I. DUFRASNE
}

Université de Liège, Faculté de Médecine vétérinaire, Département des Productions Animales, Boulevard de Colonster, B43, B-4000 Liège, Belgique

Courriel : isabelle.dufrasne@ulg.ac.be

\begin{abstract}
Une part importante de la vie des vaches laitières se passe à l'intérieur des étables. Celles-ci sont souvent équipées de logettes pour lesquelles différents aménagements sont proposés. C'est l'éleveur qui choisit ces aménagements selon ses propres contraintes et les besoins des animaux. Mais que choisiraient ces derniers s'ils avaient la possibilité de s'exprimer?
\end{abstract}

C'est à partir de la seconde moitié du vingtième siècle que les premières étables à stabulation libre pour vaches laitières ont vu le jour en Europe, la stabulation entravée étant jusqu'alors leur seule forme de logement. Ce changement, initié aux Etats-Unis, a été motivé essentiellement par la recherche de meilleures conditions de travail, en particulier lors de la traite dont il fallait diminuer la pénibilité et le temps à lui consacrer. La conception des salles de traite a permis de répondre à ces objectifs et a conduit à l'aménagement des stabulations libres. Si, aujourd'hui, l'hébergement permanent en stabulation entravée est parfois questionné en termes de bien-être animal, cet aspect n'était à l'époque pas ou très peu évoqué.

Le choix entre les étables à stabulation libre sur paille et celles à logettes a été dès le départ influencé par les disponibilités et donc le coût de la paille, variables en fonction des régions. Par exemple, en Belgique, la forte augmentation du prix de la paille au début des années 1960 favorisa d'emblée l'implantation d'étables à stabulation libre à logettes plutôt que sur paille (Martens et al 1980). Les premières offrent également souvent de meilleures conditions d'hygiène, les animaux étant amenés à se coucher sur des zones propres. Ce type de logement soumet cependant les animaux à plus de contraintes, en leur imposant l'utilisation de logettes comme zones spécifiques de repos. De plus, les couloirs de circulation constitués de béton plein ou de caillebotis représentent des sols durs pouvant être traumatisant pour les membres des animaux. Des défauts d'aménagement des logettes peuvent aboutir à une augmentation de l'incidence de divers troubles sanitaires, en particulier des boiteries, engendrer une perte de productivité et interférer négativement avec le niveau de bien-être des animaux.
Le choix du modèle de stabulation libre, sur paille accumulée ou à logettes, va aussi conditionner le type d'effluent récolté. Le premier est associé à la production de fumier, le second étant adapté à la production de lisier. La récolte de fumier reste cependant possible en stabulation libre à logettes mais, pour obtenir un fumier suffisamment consistant, il est nécessaire de prévoir un paillage d'au minimum $3 \mathrm{~kg}$ par vache et par jour (Séité et al 2012). Des paillages entre 1,5 et $3 \mathrm{~kg}$ fournissent un effluent difficile à gérer tant au stockage qu'à l'épandage.

Ayant un peu plus de cinquante ans d'existence, cette modalité d'hébergement a connu bien des évolutions, mais de nombreuses questions restent encore posées quant aux meilleurs choix à effectuer, en particulier à propos de la nature du sol des logettes, de leurs dimensions, de la conception des séparations latérales et du dispositif limitant l'avancement des animaux. L'objectif de cet article est de synthétiser les résultats de recherches visant à évaluer l'impact de l'aménagement des logettes, tout particulièrement des différents types de sols, sur le confort des vaches et sur la fréquence et la sévérité de certaines maladies.

\section{1 / Critères d'évaluation du confort des vaches}

Une évaluation globale du niveau de bien-être des animaux en ferme nécessite de prendre en compte divers critères associés aux quatre principes à respecter : une alimentation adaptée, un logement correct, des conditions d'élevage assurant une bonne santé et permettant l'expression de comportements appropriés. Ces principes ont été établis dans le cadre d'un projet européen dénommé « Welfare
Quality ${ }^{\circledR} \gg$ (Veissier et al 2010). La conception des logettes est susceptible d'influencer tout particulièrement les résultats de l'évaluation de l'aspect logement mais aussi de la santé. Les critères retenus au sein du projet Welfare Quality ${ }^{\circledR}$ pour l'évaluation de l'aspect logement sont : le confort autour du repos, le confort thermique et la facilité de déplacement ; ceux relatifs à l'aspect santé sont : l'absence de blessures, de maladies et de douleurs causées par les pratiques d'élevage (Veissier et al 2010). En fonction des diverses catégories d'animaux de ferme, des critères spécifiques ont été définis permettant le calculer un score de bien-être à l'échelle d'un troupeau. Parmi ceux proposés pour les troupeaux laitiers et susceptibles d'être influencés par la conception des logettes, on peut retenir l'état de propreté des vaches, l'utilisation de l'aire de repos et le temps mis à se coucher - tout trois associés au confort de repos - et la proportion d'animaux présentant des signes de boiteries, des lésions corporelles ou des troubles sanitaires (dont les mammites) - ces éléments étant utilisés pour l'évaluation de la santé - (Botreau et al 2009, Coignard et al 2013).

Si les critères définis ci-dessus sont adaptés à une évaluation globale, réalisée en ferme, du bien-être d'un troupeau, l'observation prolongée et/ou détaillée des animaux, en conditions expérimentales, permet d'ajouter d'autres paramètres. Par exemple, on peut prendre en compte comme indicateurs du confort de couchage, la durée et la fréquence de couchage, la durée moyenne d'une période de repos ainsi que la facilité avec laquelle l'animal se couche et se relève.

Lorsqu'ils se couchent ou se relèvent, les bovins passent par une position à genoux (figure 1). A ce moment, ceux-ci 
supportent plus de la moitié du poids du corps et la souplesse du sol va inévitablement conditionner le confort des animaux. Des sols durs peuvent les amener à se coucher moins souvent, cette réduction pouvant partiellement être compensée par un allongement de la durée moyenne des périodes couchées (Rushen et al 2007). En pratique, si une personne qui se laisse tomber à genoux sur une surface perçoit une douleur, il devrait en être de même pour un bovin (Vin et VinDekoker 2007). Lorsqu'ils se couchent ou se relèvent, les bovins présentent un mouvement d'extension de la tête et du cou vers l'avant. Une vache couchée a besoin d'un espace d'au moins $70 \mathrm{~cm}$ devant elle pour réaliser cette extension.

Le temps consacré au mouvement de coucher peut servir d'indicateur de la facilité avec laquelle la vache peut l'exécuter. En prairie ou sur litière accumulée, le temps s'écoulant entre le début de la flexion des genoux et la position couchée est de l'ordre de 7 à 8 secondes alors que pour des vaches en stabulation entravée sur sol bétonné il atteint 14 secondes. De même, des vaches entravées interrompent le mouvement de flexion des genoux associé au couchage deux fois plus souvent que des vaches qui ne sont pas attachées (Krohn et Munksgaard 1993).

La durée de couchage des vaches laitières est de l'ordre de $12 \mathrm{~h} / 24 \mathrm{~h}$ et elle peut varier en fonction des conditions de stabulation entre 8 et $16 \mathrm{~h} / 24 \mathrm{~h}$ (Tucker et al 2009). Haley et al (2000) ont relevé que des vaches attachées sur un bâti en béton réduisent de plus de $4 \mathrm{~h}(10,5 \mathrm{vs}$ $14,7 \mathrm{~h}$ ) leur temps de couchage par rapport à celui observé pour des vaches en box individuel $\left(16 \mathrm{~m}^{2}\right)$ avec un sol couvert d'un matelas. Des écarts de même ampleur $(4 \mathrm{~h} / \mathrm{j})$ ont été rapportés par plusieurs études comparant les conditions de confort en stabulations libres à logettes (Tucker et al 2006).

La nombre quotidien de changements de position (couchés vs debout et vice versa) varie entre 10 et 15 et la durée moyenne d'une période couchée entre 60 et 80 minutes (CIGR 1994). Ces mouvements sont conditionnés par la succession des diverses activités journalières (alimentation, traite) et sans doute aussi par le besoin que ressent l'animal de changer de position afin d'éviter des excès de pression sur diverses régions du corps (Tucker et al 2009). Ces changements de position sollicitent les diverses articulations des membres, en particulier les genoux et les jarrets. En règle générale, les animaux présentant une boiterie clinique ont tendance à être moins souvent couchés (Galindo et al 2000, Cook et al 2005), ce qui laisse supposer que si les mouvements associés
Figure 1. Schémas de déplacement du corps lors des comportements de lever et de coucher (adapté de Lidfors 1989).
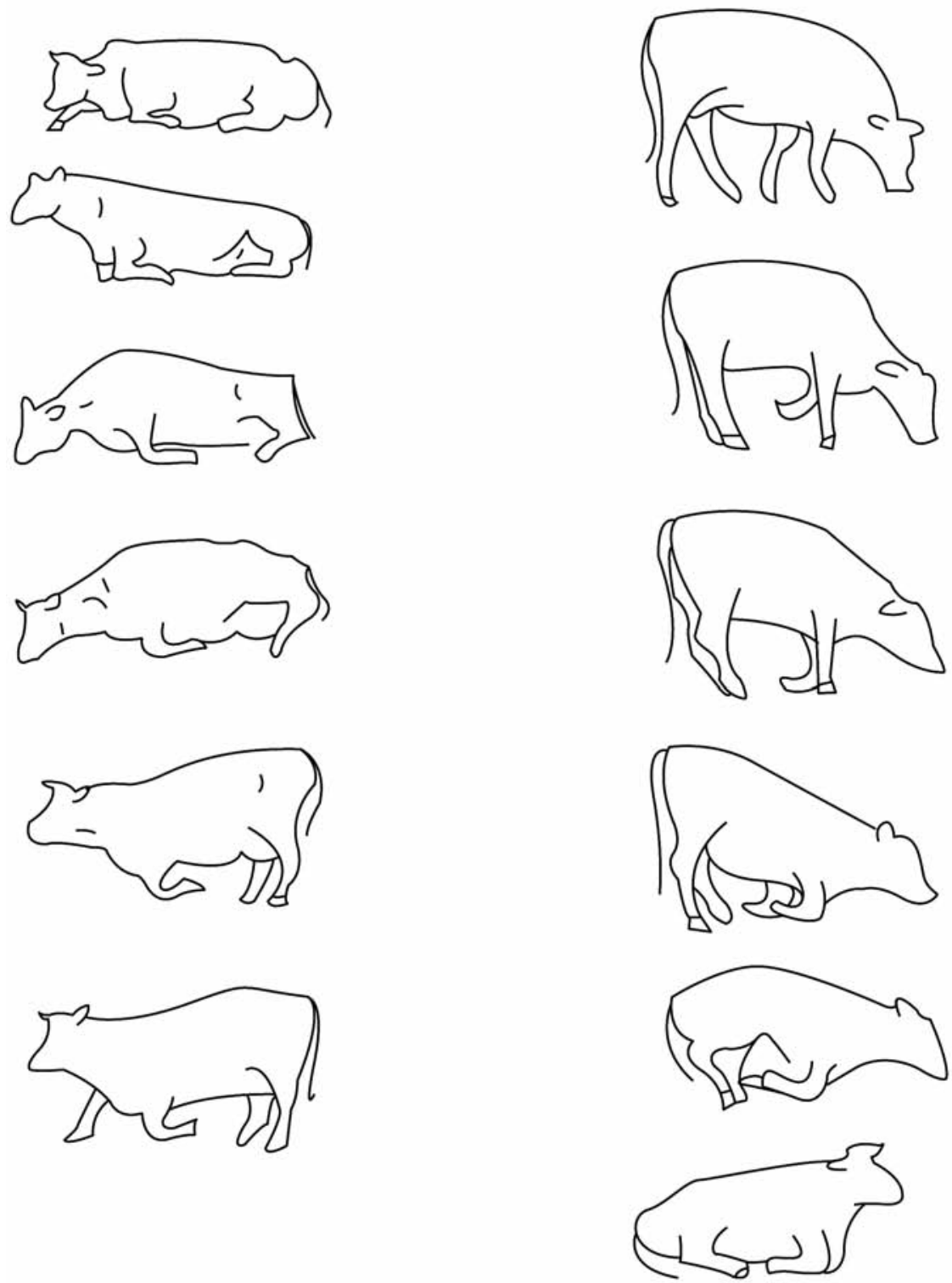

au coucher et au lever sont douloureux, ils préfèrent rester plus longtemps debout. Cette situation peut engendrer un cercle vicieux car la station debout peut retarder la guérison d'atteintes des articulations et des pieds. Des corrélations significatives ont été mises en évidence entre d'une part, les types de sol et, d'autre part, la fréquence des boiteries et leur sévérité (Tucker et al 2009), chiffrable à partir de grilles d'évaluation de la démarche des animaux (cf. encadré).

Pour les vaches laitières hébergées dans les étables à stabulation libre à logettes, Cook et al (2005) ont défini un index de confort correspondant au pourcentage de vaches couchées dans les logettes par rapport aux vaches en contact avec celles-ci (couchées, debout avec soit les 2 membres antérieurs ou les 4 membres sur le bâti). Cet index devrait être supérieur à $80 \%$ et un pourcentage de vaches debout supérieur à $20 \%$ est à considérer comme indicateur d'un problème de boiterie.

Notons que l'interprétation des observations réalisées sur des animaux placés en situation de choix ou auxquels des changements sont proposés doit tenir compte du fait que leur comportement face à des situations nouvelles est en partie conditionné par leur expérience antérieure. Souvent, ils gardent au départ une préférence pour la situation qu'ils connaissent même si celle-ci s'avère in fine moins confortable (Manninen et al 2002, Tucker et al 2003, Norring et al 2008). Plusieurs mois seraient nécessaires pour habituer des vaches à de nouveaux types de sol. Les tests comparatifs plaçant les animaux dans une situation de choix doivent donc comprendre une période d'habituation suffisamment longue et tout changement important imposé 


\section{Encadré. Evaluation de la sévérité des boiteries des vaches laitières.}

La fréquence de boiteries dans un troupeau peut être utilisée comme un des critères d'évaluation du bien-être. La présence de boiteries peut être objectivée à l'aide de grilles de notes (Hulsen 2006). Les observations doivent être faites régulièrement sur chaque vache, idéalement une fois par mois, et les résultats conservés afin de pouvoir en contrôler l'évolution. Les observations sont à réaliser sur des animaux qui marchent naturellement sur une surface plate. L'objectif est qu'il y ait, à un moment donné, moins de $5 \%$ de vaches présentant les scores les plus élevés. La grille de notation de Hulsen (2006) est reprise ci-dessous.

Note 1 : La démarche est stable et normale. La vache se tient debout, pose ses pieds sans hésitation. Elle pose les pieds arrière à l'endroit où étaient les pieds avant. Le dos est plat et horizontal au repos comme en mouvement.

Note 2 : La démarche est légèrement anormale. A l'arrêt, le dos est plat et horizontal tandis qu'en mouvement, le dos est courbé. La tête est tenue plus éloignée et plus basse que le corps.

Note 3 : A l'arrêt comme en mouvement, le dos est légèrement courbé. La vache fait de petits pas avec un ou plusieurs membres.

Note 4 : La vache boite franchement et soulage un ou plusieurs membres. Le dos est courbé à l'arrêt comme en mouvement.

Note 5 : La vache boite gravement, se lève difficilement, est souvent couchée. Elle évite de mettre du poids sur un de ses membres.

aux animaux devrait idéalement prévoir une phase d'adaptation. L'interprétation d'observations comportementales doit également prendre en compte le fait que le choix d'un individu peut être influencé par son statut hiérarchique. Par exemple, des vaches peuvent préférer une situation de repos moins confortable à une trop grande proximité de congénères de statut hiérarchique supérieur.

Enfin, concernant le lien entre le temps passé en position couchée et le niveau de production laitière, il a été démontré que le débit sanguin vers le pis augmente d'un peu plus de $25 \%$ lorsque les vaches sont couchées plutôt que debout (Rulquin et Caudal 1992, Metcalf et al 1992). La synthèse de lait est donc favorisée par la position couchée.

\section{2 / Les caractéristiques des types de sol}

Trois types de sol doivent être distingués :

- les sols bétonnés recouverts ou non d'un tapis ou d'un matelas ;

- les sols recouverts d'une couche épaisse $(15$ à $20 \mathrm{~cm})$ de litière organique ;

- les sols recouverts d'une couche épaisse de sable.

Ils peuvent être comparés sur la base du niveau de confort des vaches, selon des critères définis ci-dessus mais aussi quant à leur impact sur la propreté du pis, la santé du pis, l'hygiène de la traite et la qualité du lait ainsi que sur leur coût d'installation et d'entretien.

L'état de propreté du pis est fortement tributaire de l'état de propreté du sol, variable en fonction du type choisi mais également de son niveau d'entretien. Des grilles d'évaluation de l'état de propreté des mamelles ainsi que de la partie distale des membres postérieurs des vaches sont disponibles (Hulsen 2006). Notons que plus les matières fécales sont liquides et plus les vaches sont sales. Aussi, est-il important de repérer des anomalies de consistance des matières fécales.

Les coûts d'installation des sols des logettes peuvent varier du simple à plus du double et il en va de même des coûts d'entretien qui dépendent du type et des quantités de litière requises et du temps nécessaire pour le maintien d'un bon état de propreté du bâti.

\section{1 / Sols bétonnés recouverts ou non de tapis ou de matelas}

Il ressort de plusieurs études que les vaches préfèrent les sols souples aux sols durs, c'est-à-dire ceux recouverts de tapis ou de matelas comparés à des sols bétonnés qui ne sont recouverts que d'un peu de litière (Haley et al 2000 et 2001, Tucker et Weary 2001). En situation de choix, $80 \%$ des positions couchées ont été observées sur tapis et seulement $20 \%$ sur du béton paillé (Norring et al 2010). Notons que des essais réalisés en Finlande (Manninen et al 2002) ont montré des différences de préférence en fonction de la saison, les vaches préférant se coucher sur des bâtis paillés plutôt que sur des tapis en saison hivernale, alors que la répartition des couchers se faisait à parts égales entre les deux types de sols en saison estivale; dans ce cas, on peut supposer que le choix des vaches était influencé par le niveau de confort thermique associé aux deux types de sol.
Les tapis sont normalement constitués d'un seul et même matériau (caoutchouc ou vinyle multicouche) de 1,5 à $3 \mathrm{~cm}$ d'épaisseur. Quant aux matelas, ils sont constitués d'une enveloppe en toile étanche ou en caoutchouc contenant un matériau de confort (billes de caoutchouc, mousse de polyéthylène, substance semiliquide, eau...); ils sont plus épais que les tapis et donc plus souples. A ce propos, Ruud et al (2010) distinguent les tapis des matelas en fonction de l'épaisseur de la couche déformable (déterminée au laboratoire) qui varie de 1 à $8 \mathrm{~mm}$ pour les tapis et est supérieure à $8 \mathrm{~mm}$ pour les matelas. Au sein des diverses marques commerciales de matelas, ces auteurs ont répertorié trois catégories en fonction de cette épaisseur : de 9 à $16 \mathrm{~mm}$ (catégorie 1), de 17 à $24 \mathrm{~mm}$ (catégorie 2) et supérieure à $24 \mathrm{~mm}$ (catégorie 3 ). Il ressort de leur enquête que la production laitière est plus élevée dans les étables où les vaches disposent de logettes équipées de matelas de catégories 2 et 3 comparativement aux étables équipées de matelas de catégorie 1 et, a fortiori, de tapis. De plus, l'incidence des mammites au sein des troupeaux disposant de matelas de catégories 2 et 3 était inférieure à celle des troupeaux hébergés en logettes sur sols bétonnés paillés, ces derniers étant associés à l'incidence la plus élevée de lésions des trayons. Concernant la préférence des vaches, le caractère souple (moelleux) du revêtement des logettes apparaît déterminant.

D'autres critères que la souplesse doivent être pris en compte dans la comparaison de divers modèles de matelas, dont le caractère antidérapant de la surface, la résistance au frottement et au nettoyage à haute pression, sans oublier le coût par emplacement qui dépend de la durée de vie du matériau, de la facilité d'installation et de celle des éventuelles réparations. Concernant le coût d'installation, la mise en place de tapis ou de matelas représente des suppléments de respectivement 40 et $100 \%$ par place par rapport à un béton nu (Séité et al 2012).

Il a également été proposé d'épandre une couche d'asphalte sur le béton. L'asphalte est moins glissant et assure un meilleur confort thermique, mais sa mise en œuvre, par des entreprises spécialisées, représente un surcoût de $45 \%$ par rapport à du béton nu (Séité et al 2012). La réalisation du bâti en enrobé bitumeux passe aussi par l'intermédiaire de spécialistes pour un revêtement dont 1'abrasivité est supérieure à celle du béton.

L'utilisation de tapis ou de matelas ne permet pas de renoncer à l'utilisation de litière dont le rôle est d'absorber l'humidité (urine, lait...) en surface pour notamment éviter que le revêtement ne 
devienne trop glissant. La présence de litière influence aussi l'attrait des logettes et le temps passé en position couchée. C'est ainsi que dans les jours suivant le dépôt sur des matelas de 7,5 kg de sciure sèche, les vaches se sont couchées $1,5 \mathrm{~h}$ de plus par jour comparativement à d'autres vaches n'ayant accès qu'à des matelas sans sciure (13,8 vs 12,3 h/24 h), les nombres de périodes couchées étant respectivement de 10 et 8,5/24 h (Tucker et Weary 2004). Lors de choix entre ces deux situations, plus de $85 \%$ des temps couchés sont relevés sur les logettes avec sciure.

Le choix du matériau de litière peut avoir une incidence sur la population bactérienne se développant au sein de celle-ci et par voie de conséquence sur les trayons. Des différences tant quantitatives que qualitatives entre populations bactériennes peuvent éventuellement se répercuter sur la fréquence des mammites et sur la teneur en cellules du lait. Les litières dites inorganiques (sable) se prêtent nettement moins à la prolifération bactérienne que les litières organiques (paille, sciure, copeaux de bois...). Cependant, dès qu'elle est souillée par les déjections, une litière inorganique tend à perdre cet avantage. A titre indicatif, une règle empirique précise que le seuil de contamination bactérienne de la litière, au-delà duquel le risque de mammite est accru, se situe à 1 million d'unités formant colonie par gramme de produit frais, après étalement d'un échantillon sur milieu de culture (Milklait 2013).

De la sciure « fraiche », non contaminée par les déjections peut contenir des quantités non négligeables de bactéries gram négatif, en particulier de Klebsiella et il ressort de recherches menées au NouveauBrunswick (Canada) que cette litière doit être évitée dans les troupeaux présentant des mammites à Klebsiella, en étant remplacée par de la paille ou du sable. En revanche, la paille devrait être évitée et idéalement remplacée par du sable pour les troupeaux touchés par la mammite streptococcique (MilkLait 2013).

Il ressort d'une étude de Kristula et al (2008) que, comparativement à l'utilisation de copeaux de bois ou de cendres de charbon ( $1 \mathrm{~kg}$ tous les deux jours par logette dans les 2 cas), l'utilisation de chaux hydratée $(0,5 \mathrm{~kg}$ tous les deux jours par logette) assure une réduction du nombre de bactéries (coliformes, Streptococcus spp., Klebsiella spp., Escherichia coli), par gramme de litière. Comparées à des vaches « contrôle » dont l'aire de couchage était sans litière, celles ayant accès aux logettes couvertes de chaux présentaient des trayons significativement moins contaminés par les coliformes et Klebsiella spp. Notons, cependant, que l'utilisation de chaux à la dose précitée a provoqué une irritation de la peau au niveau du pis et des jambes des vaches. La chaux hydratée devrait être utilisée à la dose d'entretien de $0,125 \mathrm{~kg}$ par logette et par jour après utilisation d'une dose initiale comprise entre 0,25 et $0,37 \mathrm{~kg}$ (Milklait 2013) ; dans tous les cas, la chaux devrait être recouverte de paille pour éviter un contact direct avec la peau du pis.

$\mathrm{Au}$ sein des exploitations laitières qui se sont équipées pour effectuer une séparation des effluents en deux fractions, liquide et solide, cette dernière est parfois réutilisée comme matériau de litière. Pour réduire le risque de transmission de microorganismes pathogènes ce fumier séché peut être composté. Il est utilisé en couverture de tapis ou de matelas ou en litière profonde. A ce jour, peu d'informations sont disponibles sur les conséquences à long terme de l'utilisation de ce type de litière. Les quelques résultats rapportés en provenance notamment de Suisse (Schrade et Zähner 2008), d'Italie (Ferrari et Moscatelli 2009), du Nouveau-Brunswick (Milklait 2013), ne signalent pas de problèmes spécifiques en termes de confort des animaux, d'impact sur leur état de santé ou sur la qualité du lait.

Plus que le choix d'un matériau de litière, il semble que sa gestion, en particulier la fréquence de renouvellement des parties souillées par de la litière propre, représente le facteur qui a le plus d'impact sur son niveau de contamination bactérienne. Dodd et al (1984) ont montré qu'un entretien journalier de la partie arrière des logettes (sur environ $1 \mathrm{~m}^{2}$ ) réduisait très significativement le nombre de coliformes par gramme de litière à base de sciure, comparativement à un entretien hebdomadaire.

L'équipement des couloirs des étables à logettes avec des revêtements en caoutchouc présente aussi des avantages. Il ressort d'une synthèse réalisée sur le sujet (Lensink et Mounier 2012) qu'ils permettent un meilleur appui pour les vaches, ce qui améliore notamment l'expression des chaleurs. La moindre usure des onglons sur ces sols demande cependant de mettre en place un programme régulier de parage.

\section{2 / Sols recouverts d'une couche épaisse de litière organique}

Si lors de l'utilisation de tapis ou matelas, il est prévu de surélever le bâti d'une vingtaine de centimètres par rapport aux couloirs de circulation, il est également envisageable de prévoir un sol de logettes à même niveau que le couloir de circulation dont il est alors séparé par une traverse d'une vingtaine de centimètres de hauteur. Cet aménagement de logettes dites « creuses » permet de maintenir sur le bâti une couche épaisse de litière ; il nécessite un investissement de $40 \%$ inférieur à celui requis pour des logettes « classiques » mais le travail d'entretien est inévitablement plus conséquent. La figure 2 (Séité et al 2012) montre la

Figure 2. Conception de logettes à sol en béton couvert de matelas, de tapis ou de litière et de logettes " creuses » remplies d'une couche épaisse de litière (adapté de Séité et al 2012).
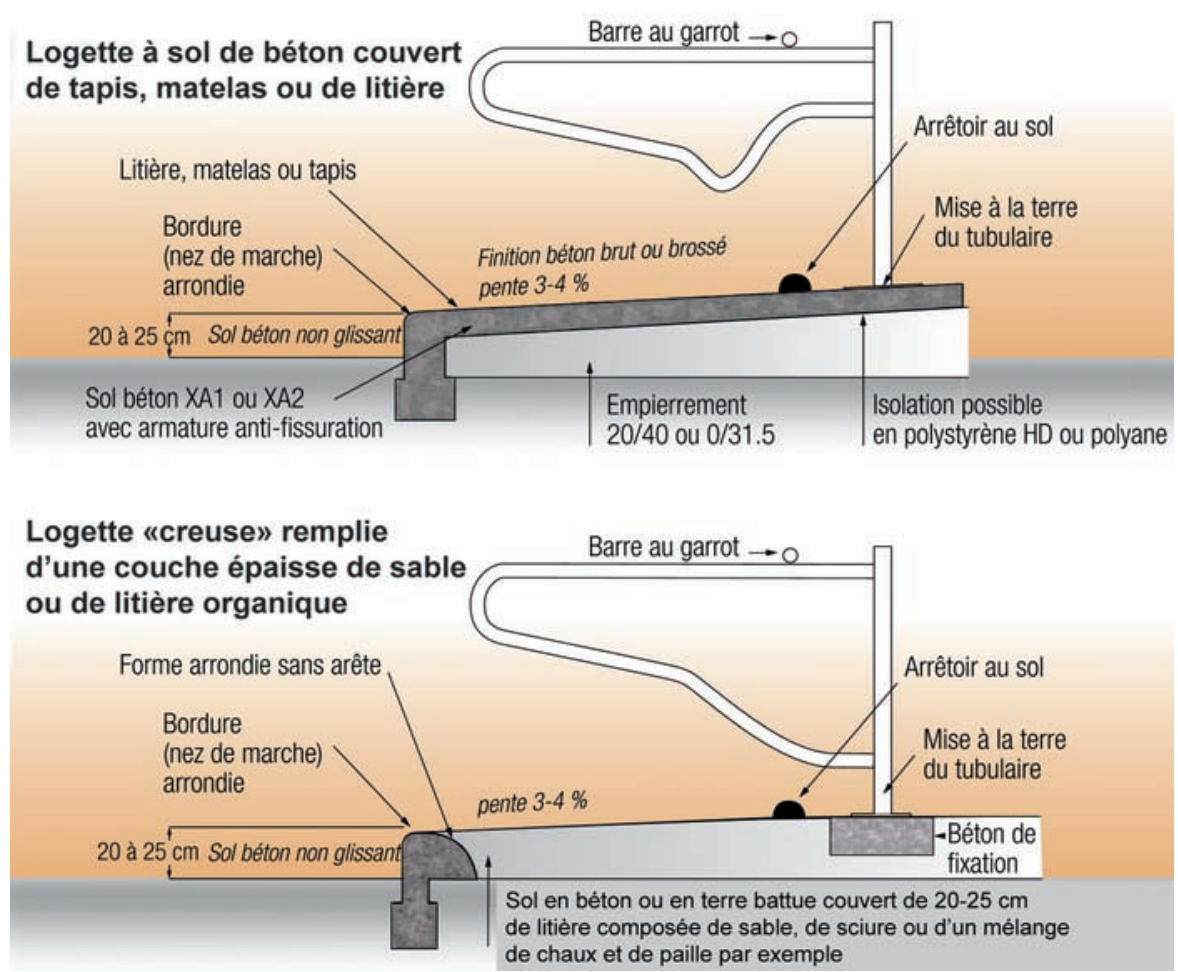
différence d'aménagement du sol de ces deux types de logettes.

En situation de choix, les vaches montrent une nette préférence pour des logettes présentant une litière épaisse de sciure $(20$ à $30 \mathrm{~cm})$ comparativement à celles équipées de matelas recouverts de 2 à $3 \mathrm{~cm}$ de sciure (Tucker et al 2003). Il ressort également d'une comparaison effectuée par Cook et al (2005) entre six troupeaux disposant de logettes avec litière épaisse de sable et six autres disposant de logettes avec matelas que la prévalence des boiteries était trois fois moins importante au sein du premier groupe $(8,2$ vs 29,3\%), les index de confort, à savoir les pourcentages de vaches couchées dans les logettes par rapport aux vaches en contact avec cellesci (cf. § 1) étant respectivement de 0,86 et 0,76 .

La litière peut être réalisée par association de paille et de chaux humide, cette dernière ayant un pouvoir asséchant et assainissant. En pratique, de la paille, hachée en brins de 3 à $4 \mathrm{~cm}$, est humidifiée dans une mélangeuse $(2,25 \mathrm{~L}$ d'eau $/ \mathrm{kg}$ de paille) afin que la chaux y adhère bien, puis celle-ci est ajoutée à raison de $6 \mathrm{~kg} / \mathrm{kg}$ de paille. Pour assurer l'entretien, un rechargement est recommandé toutes les trois semaines avec un mélange deux fois moins humide et deux fois moins chargé en chaux. Eventuellement, le remplissage peut se faire uniquement avec de la paille au cours d'une période qui ne devrait pas dépasser trois mois. Par logette, selon Capdeville (communication personnelle), il faut prévoir au départ $8 \mathrm{~kg}$ de paille et $48 \mathrm{~kg}$ de chaux et pour l'entretien $3 \mathrm{~kg}$ de mélange par jour $(0,75 \mathrm{~kg}$ de paille et $2,25 \mathrm{~kg}$ de chaux).

Aux Etats-Unis, Husfeldt et Endres (2012) ont réalisé une étude au sein de 34 exploitations laitières équipées de logettes dont le sol était recouvert soit d'une litière de fumier solide recyclé (22,1 cm d'épaisseur), soit de matelas eux-mêmes recouverts de fumier recyclé d'une épaisseur moyenne de $9,1 \mathrm{~cm}$. Au sein de ces deux groupes, les fréquences de boiteries ont été respectivement de 14 et $20 \%$ et celles de lésions des jarrets de 49 et $63 \%$. En revanche aucune différence n'a été relevée quant au niveau de production laitière et à la prévalence de mammites.

\section{3 / Sols recouverts de sable}

Il ressort d'une étude menée par Norring et al (2008) que, contrairement à ce que l'on aurait pu attendre, les vaches testées, ne présentaient pas de préférence particulière pour un sol de logettes couvert de sable (20 cm d'épaisseur) comparativement à un sol bétonné paillé $(0,7 \mathrm{~kg}$ de paille $/ \mathrm{j}$ après un apport initial de $6,5 \mathrm{~kg}$ ).
Néanmoins, le sable a permis de maintenir les vaches plus propres et elles ont présenté moins de lésions au niveau des jarrets. Un essai antérieur mené par la même équipe de recherche (Manninen et al 2002), et plaçant les animaux en situation de choix, avait par ailleurs montré une occupation nettement plus faible de logettes couvertes de sable $(20 \mathrm{~cm}$ d'épaisseur) comparativement à des logettes paillées ou équipées de tapis. La nonhabituation des vaches au sable a été évoquée pour expliquer ce résultat.

Contrairement aux observations rapportées ci-dessus, Calamari et al (2009), en proposant aux vaches un choix entre quatre types de revêtement de sol de logettes, ont relevé que celles couvertes de sable étaient les plus occupées, le temps de couchage se répartissant à $44 \%$ sur le sable (20 cm d'épaisseur), 33\% sur sol couvert de paille, $12 \%$ sur tapis et $11 \%$ sur matelas (20 cm d'épaisseur). Il est à noter que ces observations ont été réalisées 7 mois après la mise à disposition des vaches de l'ensemble de l'installation, ce qui a permis aux animaux, disposant au préalable de logettes à sol couvert de paille, de s'y habituer. Les mêmes auteurs ont également noté qu'entre 4 groupes de vaches disposant chacun, en permanence, d'un des 4 types de sol décrit ci-dessus, le niveau de production laitière du groupe sur sable a été supérieur à celui des 3 autres.

Notons que l'effet positif du sable sur le confort de couchage des vaches dépend de l'épaisseur prévue. Pour une différence d'épaisseur de l'ordre de $14 \mathrm{~cm}(6 \mathrm{~cm}$ au lieu de $20 \mathrm{~cm}$ ), le temps moyen couché par $24 \mathrm{~h}$ a été réduit de $2,33 \mathrm{~h}$, soit une réduction de $10 \mathrm{~min}$ par $\mathrm{cm}$ (Drissler et al 2005).

Un impact positif de sols de logettes recouverts de sable ressort d'une étude réalisée au Danemark au sein de 37 fermes laitières (Andreasen et Forkma 2012). Cette étude a en effet montré que tant la fréquence des lésions au niveau des jarrets $(3 \%$ vs $35 \%)$, que celle de gonflements des genoux (0,7 vs 7\%), d'alopécies (12 vs $73 \%$ ) et, de façon plus générale, la fréquence de boiteries (44 vs $61 \%$ ), sont nettement inférieures dans les étables utilisant du sable que dans celles dont les logettes sont équipées de tapis ou de matelas. De plus, l'état de propreté des vaches et leur niveau de production laitière y sont meilleurs. Cette étude confirme des résultats obtenus précédemment soulignant une moindre fréquence de lésions aux jarrets (Weary et Taszkun 2000) ainsi qu'une moindre prévalence des boiteries (Cook et al 2004, Espejo et al 2006) parmi les vaches disposant de logettes à sol couvert de sable comparativement à celles utilisant des logettes à sols couverts de matelas.
A propos de l'impact du matériau de litière sur le développement des populations bactériennes au sein de celle-ci et concomitamment sur les trayons, le sable en litière profonde permettrait, comparativement à des litières profondes de sciure, de réduire les populations de coliformes et de Klebsiella, mais favoriserait celle de streptocoques (Zdanowicz et al 2004). L'état de propreté de la litière de sable reste de première importance. $\mathrm{Si}$ du sable propre, en tant que matériau inorganique, ne se prête pas à la prolifération bactérienne, cet avantage se perd progressivement dès que le sable est souillé par des matières fécales et de l'urine.

$\mathrm{Si}$, du point de vue du confort animal, le sable semble incontestablement une solution de choix comme revêtement du sol des logettes, il faut savoir qu'il provoque, par son caractère abrasif, une dégradation accélérée de l'équipement de manutention des effluents (Husfeldt et Endres 2012) ainsi que du matériel des logettes (Andreasen et Forkman 2012). La manutention de fumiers (transfert, stockage, épandage) chargés de sable nécessite des équipements spécialement conçus, ceux servant à la manutention des fumiers à litière organique étant difficilement adaptables (Milklait 2013). Enfin, l'entretien de la couche, et en particulier sa mise à niveau régulière, représente une charge de travail supplémentaire. Des équipements spécifiques (lanceurs de sable) ont cependant été élaborés pour permettre une mécanisation du réapprovisionnement régulier en sable des logettes (Milklait 2013). Il a également été signalé que l'utilisation de sable s'accompagne d'une augmentation des coûts de maintenance des robots de traite (Rodriguez 2014). Les frais spécifiques à consentir pour la gestion de logettes à couche épaisse de sable seront évidemment d'autant plus rapidement amortis que la taille du troupeau est élevée.

Pour procurer à la vache un maximum de confort et assurer la propreté du bâti, il conviendrait de prévoir $20 \mathrm{~kg}$ de sable par logette et par jour, l'épaisseur optimale étant comprise entre 15 et $20 \mathrm{~cm}$. Réduire la quantité à $10 \mathrm{~kg}$ par jour affecte à la fois le confort et la propreté des vaches (Milklait 2013). La granulométrie doit être contrôlée; les grains de sable devraient avoir un diamètre compris entre 1 et $2 \mathrm{~mm}$; un sable grossier peut occasionner des écorchures, en particulier au niveau des genoux et un sable extrafin aura tendance à s'agglomérer dans les logettes et à coller au pis.

Afin de réduire la quantité de sable à utiliser, il a été proposé d'associer l'utilisation de matelas et de sable. Cependant, cette solution est plus coûteuse à l'installation. Pour une utilisation de sable 
en revêtement sur une épaisseur de $5 \mathrm{~cm}$, avec évacuation quotidienne du substrat souillé situé sur le 1/3 arrière des logettes, il convient de prévoir un apport de 10 à $15 \mathrm{~kg}$ de sable frais par logette et par jour (Milklait 2013). Il ressort d'une comparaison de l'occupation de logettes équipées de matelas recouverts soit d'un peu de sciure $(<1 \mathrm{~cm})$ ou de 5 à $8 \mathrm{~cm}$ de sable, que ces dernières apparaissent comme plus confortables. En effet, bien que les temps passés en position couchée n'aient pas présenté de différences significatives en fonction du type de litière, le temps moyen d'une position couchée a été supérieur sur litière de sable (79vs $57 \mathrm{~min}$ ) et les temps passés debout dans les logettes inférieurs (Cook et al 2008).

En conclusion, l'utilisation de sable comme matériau de litière pour logettes creuses dans les stabulations à logettes a été présentée dès 2009 comme allant permettre des améliorations notables de la santé, du bien-être et des performances des vaches laitières (Cook 2009). Si des résultats d'études postérieures (Andreasen et Forkman 2012) vont dans le même sens, des problèmes spécifiques liés à l'utilisation de ce matériau ont été signalés, tels que la charge plus importante de travail, des dégradations accélérées des équipements de manutention de l'effluent, l'augmentation du coût de maintenance des robots de traite. Il en résulte que le retour sur investissement du choix de ce type de sol n'est pas garanti (Rodriguez 2014). Des questions restent également posées à propos du devenir du sable souillé.

\section{4 / Récapitulatif des paramètres comparatifs des principaux types de sol de logettes}

Le tableau 1 présente une comparaison des principaux types de sol quant au niveau de confort qu'ils procurent aux animaux, leur facilité d'entretien et leur coût. Les sols en béton recouverts ou non de tapis obtiennent les plus faibles scores en termes de confort et devraient être à ce titre évités lors de nouvelles constructions. Un bon niveau de confort est atteint avec les matelas mais il reste inférieur à celui des logettes creuses (avec litière); le résultat de la comparaison est cependant tributaire de la souplesse du matelas variable en fonction des marques. Le meilleur score en termes de confort est obtenu avec les logettes creuses, celles à base de sable ayant vraisemblablement un avantage sur celles à base de litière organique. De plus, le coût d'installation des logettes creuses est inférieur à celui de celles équipées de matelas, mais les premières demandent plus de temps à consacrer journellement à leur entretien indispensable au maintien d'un bon niveau de confort.

\section{3 / Les dimensions et le nom- bre de logettes}

La largeur et la longueur des emplacements doivent délimiter un espace où l'animal peut se coucher et se relever sans difficulté et se reposer à son aise. Plus cet espace est grand et meilleur devrait être le confort de l'animal, mais un excès de largeur ou/et de longueur peut cependant être globalement contreproductif car, en ne limitant pas l'espace que l'animal peut occuper, on lui permet de salir le bâti avec ses déjections et de créer ainsi des conditions favorisant la souillure du pis, préjudiciable à l'hygiène de la traite et favorisant l'apparition de mammites.

$\mathrm{Si}$, en théorie, on précise que les dimensions du bâti doivent être adaptées au gabarit des animaux, il est un fait qu'au sein d'un troupeau, tous les animaux n'ont pas le même gabarit et qu'inévitablement certains d'entre eux ne trouveront pas « chaussures à leur pied ». Des correspondances entre poids des vaches, Hauteur au garrot $(\mathrm{H})$, Longueur $(\mathrm{L}$ : prise en diagonale de la pointe de l'épaule à la pointe de la fesse) et largeur (1: mesurée au niveau des épaules) ont été proposées (tableau 2). Ces valeurs permettent d'obtenir des estimations de largeur ( $\left(l_{\text {logette }}\right)$ et de longueur $\left(\mathrm{L}_{\text {logette }}\right)$ de logettes adaptées au gabarit des animaux. Pour la longueur, il convient de différencier les logettes face à un mur des autres. Les remières nécessitent plus d'espace pour permettre à l'animal de pencher le corps vers l'avant lorsqu'il se relève.

L'application de ces formules à des vaches de $750 \mathrm{~kg}$ donne une largeur de logette de $1,18 \mathrm{~m}$ et des longueurs de respectivement 2,55 et $2,21 \mathrm{~m}$.

La tendance actuelle est de recommander des longueurs plus grandes, à savoir pour des logettes face à un mur, jusqu'à $2,70 \mathrm{~m}$ (vaches de $800 \mathrm{~kg}$ ) (The Dairy Group 2012), voire 2,80 m (hauteur au garrot de 1,47 m) (Séité et al 2012) et même $3,05 \mathrm{~m}$ (vaches de $820 \mathrm{~kg}$ ) (Cook 2009). Pour des logettes en face à face et pour des animaux de même gabarit que ci-dessus, les recommandations vont de 2,40 m (Séité et al 2012, The Dairy Group 2012) à 2,59 m (Cook 2009). Notons que, en disposition face à face imposant un partage d'un espace antérieur commun, le mouvement de lever d'une vache peut induire le lever de celle qui lui fait face. Concernant la largeur des logettes, la plupart des recommandations actuelles la fixent à 1,2-1,25 m (Séité et al 2012, The Dairy Group 2012).

Une pente de 2 à $3 \%$ de l'avant vers l'arrière encourage les animaux à se positionner la tête vers l'avant et facilite l'évacuation des liquides (urine, eau de nettoyage) (The Dairy Group 2012). Des pentes supérieures sont déconseillées parce qu'elles accroissent le risque de glissances, la perte de litière et les efforts lors du relevé (Séité et al 2012).

Tableau 1. Comparaison des principaux types de sol de logettes sur la base $d u$ niveau de confort des animaux, de la facilité d'entretien et du coût par place.

\begin{tabular}{|c|c|c|c|}
\hline & Niveau de confort & Facilité d'entretien & Coût* \\
\hline Béton + litière & - & + & 100 \\
\hline $\begin{array}{l}\text { Béton + tapis } \\
\text { (avec litière) }\end{array}$ & + & ++ & 140 \\
\hline $\begin{array}{l}\text { Béton + matelas } \\
\text { (avec litière) }\end{array}$ & ++ & ++ & 200 \\
\hline $\begin{array}{l}\text { Logettes creuses } \\
\text { Litière organique }\end{array}$ & +++ & - & 60 \\
\hline $\begin{array}{l}\text { Logettes creuses } \\
\text { Litière de sable }\end{array}$ & $+++(+)$ & - & 85 \\
\hline
\end{tabular}

${ }^{*}$ : hors matériaux de litière - indice $100=150 €$ en 2012 (Séité et a/ 2012).

Tableau 2. Relations entre le Poids des vaches $(P)$, la Hauteur au garrot $(H)$, la Longueur ( $L$ : de la pointe de l'épaule à la pointe de la fesse) et la largeur ( $\mathrm{l}$ : au niveau des épaules) (CIGR 1994).

\begin{tabular}{|c|c|c|c|}
\hline Poids (kg) & Hauteur (m) & Longueur (m) & Largeur $(\mathbf{m})$ \\
\hline 550 & 1,35 & 1,61 & 0,50 \\
\hline 650 & 1,39 & 1,69 & 0,55 \\
\hline 750 & 1,42 & 1,75 & 0,60 \\
\hline
\end{tabular}

Les relations suivantes ont été proposées pour calculer les dimensions des logettes (CIGR 1994) :

logette $=0,83 \mathrm{H}$.

Llogette (face à un mur) $=0,92 \mathrm{~L}+0,15+0,56 \mathrm{H}$.

$\mathrm{L}_{\text {logette }}$ (absence d'obstacle à l'avant) $=0,92 \mathrm{~L}+0,15+0,32 \mathrm{H}$. 
Les normes relatives à la dénivellation entre le bâti des logettes et le couloir de circulation arrière se situent entre 15 et $20 \mathrm{~cm}$ (CIGR 1994, Cook 2009, The Dairy Group 2012). Si la différence de niveau est inférieure à $15 \mathrm{~cm}$, il n'est pas exclu que certaines vaches puissent pénétrer en marche arrière dans les logettes et, d'autre part, si le nettoyage des couloirs se fait par raclage, des déjections pourraient venir souiller l'arrière du bâti. Une différence de niveau supérieure à $20-25 \mathrm{~cm}$ aura pour conséquence d'exercer un surcroît de pression sur les onglons arrière des vaches qui se tiennent debout avec les membres avant sur le bâti et les membres arrière dans le couloir. Afin de limiter le risque de tarsites, l'arête sera arrondie, non abrasive.

Quant au rapport entre le nombre de logettes et le nombre de vaches dans l'étable, il ressort de la revue de la littérature (The Dairy Group 2012) que la recommandation la plus fréquente est de prévoir une logette par vache et même, compte tenu du fait que dans une étable certains emplacements sont moins attractifs, par exemple ceux en bouts de rangées, d'en prévoir $5 \%$ de plus. Ceci évite aux vaches situées au bas de la hiérarchie du troupeau de rester debout dans les couloirs de circulation durant des périodes anormalement longues, avec pour conséquence une incidence plus élevée chez ces animaux de lésions des pieds.

\section{4 / Les types de séparations latérales et de délimitations à l'avant des logettes}

\section{1 / Les séparations latérales}

Les modèles actuels de séparations latérales se différencient de ceux des années 1960 par une meilleure prise en compte :

- des mouvements de tête et d'encolure que l'animal doit pouvoir effectuer pour se relever ;

- des risques de contusions dus aux frottements répétés de zones du corps, par exemple de l'angle de la hanche, sur les séparations ;

- de la gêne que ces séparations peuvent occasionner lors du nettoyage du bâti.

Parmi les séparations proposées actuellement sur le marché, Séité et al (2012) distinguent celles qui présentent une barre inférieure en partie basse permettant un appui latéral pendant le coucher, de celles, plus récentes, qui en dégageant totalement la partie basse, sont moins contraignantes pour les vaches, mais augmentent le risque de les voir se placer en diagonale et d'empiéter sur les logettes voisines. Les auteurs présentent les avantages et inconvénients des divers modèles dont deux se distinguent par la bonne contention latérale des animaux et un bon rapport qualité prix : il s'agit du modèle avec fixation de la séparation sur poteau avant individuel et de celui dit monobloc avec deux pieds de fixation à l'avant.

\section{2 / Les délimitations à l'avant}

L'avancement de la vache sur le bâti est contrôlé par une barre dite de garrot (ou de cou), positionnée transversalement sur ou au-dessus des séparations latérales. Outre la fonction de solidariser l'ensemble des logettes, cette barre a un double rôle sur le positionnement des vaches. Le premier est de les empêcher de trop s'avancer vers l'avant et, lorsque les logettes sont face à un mur, de les amener à se coucher à une certaine distance de celui-ci, leur laissant un espace libre pour pouvoir porter la tête vers l'avant lorsqu'elles se lèvent. Des vaches couchées trop près d'un mur ont des difficultés à se relever. En prairie, une vache qui s'est mise debout se trouve de 60 à $90 \mathrm{~cm}$ en avant de la place qu'elle occupait couchée (Cook 2009). Notons qu'il faut aussi éviter que les vaches se couchent trop vers l'arrière, le corps empiétant alors sur la zone correspondant au couloir de circulation et étant plus exposé à diverses contusions. Le second rôle de la barre de garrot est d'amener les vaches qui sont debout dans les logettes, à placer les membres postérieurs, juste au niveau du rebord du bâti, de telle sorte que lorsqu'elles défèquent et urinent, les déjections tombent à l'arrière du bâti.

L'emplacement de la vache debout sur la logette va être conditionné à la fois par la hauteur de la barre et la distance la séparant du rebord arrière du bâti. Si le positionnement de la barre permet à la vache de s'avancer sur le bâti plus en avant que souhaité, le sol sera plus souillé par les déjections et les pis seront plus sales. Si, au contraire, ce positionnement ne permet pas aux vaches de se tenir debout avec les membres arrière sur le bâti, d'une part, ceux-ci seront surchargés compte tenu de la dénivellation entre le bâti et le couloir de circulation et, d'autre part, les vaches seront plus longtemps debout sur les couloirs de circulation c'est-à-dire sur un sol constamment humide, ce qui peut accroître la fréquence des affections des pieds. Veissier et al (2004) suggèrent également qu'une barre de garrot, empêchant les vaches de se tenir debout avec les membres arrière sur le bâti, entraîne des temps de couchage plus longs qu'attendus, ce qui pourrait accrô̂tre le risque de contamination du pis.

Définir un positionnement idéal d'une barre de garrot qui tienne compte de l'ensemble de ces considérations et de la variabilité de gabarit des vaches au sein d'un troupeau apparaît comme une tâche irréalisable, ce qui peut expliquer la diversité des recommandations trouvées dans la littérature.

Tucker et al (2005) ont observé que le bâti de logettes sans barre de garrot était 3 fois plus souillé par les déjections des vaches $(1,3$ vs 0,5 défécations $/ 24 \mathrm{~h})$ que le bâti de logettes équipées d'une telle barre (placée à une hauteur de 124,5 cm et distante de $160 \mathrm{~cm}$ du rebord arrière de la logette). Ces mêmes auteurs ont testé 3 hauteurs $(\mathrm{H})$ de barre de garrot : 102,114 et $127 \mathrm{~cm}$ du sol ainsi que l'absence d'une telle barre. Aucune différence significative n'a été relevée pour le temps de couchage, mais les temps passés en position debout dans les logettes avec les 4 membres sur le bâti ont été respectivement de : $83 \mathrm{~min} / 24 \mathrm{~h}$ (pas de barre), $40 \min (\mathrm{H}=127 \mathrm{~cm})$ et $21 \min (\mathrm{H}=102$ ou $114 \mathrm{~cm})$. Notons que sur le temps total passé en position debout au cours d'une journée $(10,3 \mathrm{~h} / 24 \mathrm{~h}$ en moyenne lors de cette étude), la plus grande partie se passe en dehors des logettes et que, par conséquent, le confort en position debout dépend avant tout de caractéristiques qui ne sont pas en rapport avec les logettes, mais avec celles des autres endroits où les vaches se tiennent debout (couloirs de circulation, aire d'alimentation, salle de traite).

Veissier et al (2004), après avoir notamment constaté lors d'une enquête effectuée en France auprès de 70 exploitations laitières que les difficultés à se coucher ainsi que les blessures étaient plus fréquentes dans les stabulations libres à logettes équipées de barres de garrot positionnées plus haut que la moyenne, recommandent de les fixer à une hauteur équivalant à $75 \%$ de la hauteur au garrot (H). Pour la CIGR (1994), la barre devrait être située à une hauteur (h) située dans la fourchette de 75 à $85 \%$ de $\mathrm{H}$, ce qui correspond pour des vaches de $750 \mathrm{~kg}$ $(\mathrm{H}=142 \mathrm{~cm})$ à une valeur $\mathrm{h}$ comprise entre 106 et $121 \mathrm{~cm}$. Des recommandations plus récentes (Cook 2009, The Dairy Group 2012) fournissent des valeurs plus élevées, de $120 \mathrm{~cm}$ (vaches de $600 \mathrm{~kg}$ ) à $132 \mathrm{~cm}$ (vaches de $820 \mathrm{~kg}$ ).

La distance entre la barre de garrot et le rebord arrière du bâti influence également le temps passé en position debout les 4 membres sur le bâti. Pour des distances de $130 \mathrm{~cm}$ et $190 \mathrm{~cm}$, à même hauteur de barre $(125 \mathrm{~cm})$, ces temps ont été de respectivement 0 et $36 \mathrm{~min} /$ jour (Fregonesi et al 2009). Ces auteurs soulignent que cette différence était d'autant plus marquée que les vaches étaient de petites tailles, donnant l'impression que la distance de $190 \mathrm{~cm}$ était encore insuffisante pour permettre à celles de plus grande taille d'adopter cette position. En pratique, des longueurs variant entre 
$170 \mathrm{~cm}$ (vaches de $600 \mathrm{~kg}$ ) et $180 \mathrm{~cm}$ (vache de $800 \mathrm{~kg}$ ), voire jusqu'à $190 \mathrm{~cm}$, sont recommandées (Cook 2009, The Dairy Group 2012). Cook (2009) préconise de différencier ces recommandations en fonction du type de sol, les valeurs citées ci-dessus étant à retenir pour des sols équipés de matelas ; pour des logettes avec litière épaisse de sable, il prévoit une distance plus courte compte tenu de la plus grande difficulté à gérer dans ce cas les souillures à l'arrière du bâti.

En conclusion, il est important de pouvoir moduler la position de la barre de garrot, tant en hauteur qu'en profondeur (distance par rapport au seuil de la logette). Les équipements permettant ce réglage sont donc à préférer à ceux qui ne le prévoient pas.

Afin de limiter l'avancement des vaches dans les logettes et ainsi de les maintenir plus propres, il est également proposé, d'associer à la présence de la barre de garrot, un arrêtoir, placé au niveau du sol. L'arrêtoir donne un point de repère à l'animal lui indiquant qu'il est suffisamment avancé. En bois ou en matériau synthétique, fixé au bâti ou sur le bas des séparations latérales, son bord supérieur ne doit pas se situer à plus de $10 \mathrm{~cm}$ du sol (Cook 2009, The Dairy Group 2012). Une sangle textile à fixations latérales peut jouer ce rôle d'arrêtoir, pour autant qu'elle soit bien tendue. Il est généralement recommandé de prévoir une distance arrêtoir - bord arrière du bâti un rien plus courte (de $5 \mathrm{~cm}$ ) que la distance de ce bord à la barre de garrot. Cependant, ces arrêtoirs ne sont pas nécessairement appréciés par les animaux. Tucker et al (2006) ont en effet observé que les vaches passent plus de temps couchées $(+1,2 \mathrm{~h} / \mathrm{j})$ et moins de temps debout $(-22 \mathrm{~min} / \mathrm{j})$ dans des logettes sans arrêtoir que dans celles qui en sont pourvues. De même, ils ont relevé qu'en situation de choix, $68 \%$ du temps couché est passé dans des logettes sans arrêtoir. Pour Cook (2009) et Séité et al (2012), l'arrêtoir au sol reste néanmoins indispensable pour donner un repère d'avancement à la vache.

Lorsque des logettes sont positionnées face à face et que les séparations latérales sont fixées sur poteaux, des vaches, éventuellement bousculées à l'arrière, peuvent sortir par l'avant en passant sous la barre de garrot. Pour l'éviter, une barre de tête (ou une sangle bien tendue) peut être positionnée horizontalement à $1 \mathrm{~m}$ du sol à l'extrémité avant de la logette.

\section{Conclusion}

Le logement des animaux doit évidemment correspondre à leurs besoins. Il doit cependant aussi tenir compte des besoins des éleveurs et de leurs multiples contraintes, qu'elles soient financières ou en rapport avec l'organisation du travail et avec la nécessité de répondre à des normes de qualité de production.

Si les éleveurs ont fait de la stabulation libre à logettes une des principales modalités d'hébergement des vaches laitières, on peut supposer qu'elle permet de réaliser un bon compromis entre leurs contraintes et les besoins des animaux. Depuis les premières descriptions de ce type de stabulation, les recommandations relatives à sa mise en place et à sa gestion ont constamment évolué sous l'impulsion des observations des éleveurs et des résultats des recherches. Une attention particulière est accordée à l'amélioration des conditions de confort des vaches, incluant la mise en place de mesures visant à limiter les boiteries et les mammites, affections dont la fréquence et la sévérité sont dépendantes des conditions environnementales.

Les caractéristiques du sol des logettes interfèrent avec le temps passé en position couchée, la fréquence des changements de position, la facilité avec laquelle les vaches exécutent les mouvements de lever et de coucher. Il apparaît clairement des résultats des recherches que la souplesse du sol est un élément déterminant du confort, la souplesse requise étant assurée soit par des matelas, soit par des litières épaisses.

Tous les matelas n'offrent pas la même souplesse et il convient d'accorder la préférence à ceux dont la couche déformable est la plus élevée. Leur utilisation ne permet pas de se passer d'un matériau de litière dont le but premier sera d'absorber l'humidité du revêtement, tout en améliorant aussi le confort des animaux couchés. Il semble que le choix d'un matériau (paille, sciure, sable, chaux...) soit un critère moins important que la fréquence de renouvellement de la litière pour garantir la propreté du sol, du pis et en conséquence de bonnes conditions d'hygiène de traite.

Les logettes « creuses », dont le sol est recouvert d'une couche épaisse $( \pm 20 \mathrm{~cm})$ de litière, semblent globalement mieux appréciées par les vaches que celles équipées de matelas et leur utilisation est associée à une moindre fréquence de lésions au niveau des membres ainsi que de boiteries. Le sable apparaît comme un matériau de premier choix pour assurer le confort des animaux. Néanmoins, l'entretien des logettes creuses demandent un surcroît de travail et la manutention du sable comme matériau de litière nécessite des équipements spécifiques.

De nombreux autres critères que le type de sols interfèrent avec le niveau de confort des vaches dans les stabulations libres à logettes; en particulier, les dimensions des logettes et la façon de les délimiter tant latéralement qu'à l'avant. Les recommandations à ce propos entrent dans une fourchette de valeurs assez étendue car devant être adaptées au gabarit des animaux, variable en fonction des troupeaux, et intégrer la recherche de conditions optimisant la propreté du bâti.

C'est évidemment l'éleveur qui est en première ligne pour repérer d'éventuelles inadaptations des équipements d'étable à ses animaux, mais il doit pour cela avoir du temps à consacrer à leur observation. Changer les aménagements d'étables existantes dont il apparaîtrait que certaines de leurs caractéristiques ne sont plus adaptées au cheptel d'aujourd'hui n'est cependant pas toujours facile, du moins à moindre coût. Aussi, lors de nouvelles constructions, il conviendrait d'être attentif dès le départ aux possibilités de réaménagements qui s'imposeraient par la suite.

\section{Remerciements}

Les auteurs remercient Kim Brasseur pour les croquis de la figure 1 . 


\section{Références}

Andreasen S.N., Forkman B., 2012. The welfare of dairy cows is improved in relation to cleanliness and integument alterations on the hocks and lameness when sand is used as stall surface. J. Dairy Sci., 95, 4961-4967.

Botreau R., Veissier I., Perny P., 2009. Overall assessment of animal welfare: strategy adopted in Welfare Quality ${ }^{\circledR}$. Anim. Welfare, 18, 363370.

Calamari L., Calegari F., Stefanini L., 2009. Effect of different free stall surfaces on behavioural, productive and metabolic parameters in dairy cows. Appl. Anim. Behav. Sci., 120, 9-17.

CIGR, 1994. The design of dairy cow housing. Report of the CIGR section II Working group n॰14 - cattle housing, Farm buildings research team. Winchester, Hampshire, UK: ADAS Bridgets Dairy Research Centre.

Coignard M., Guatteo R., Veissier I., de Boyer des Roches A., Mounier L., Lehébel A., Bareille N., 2013. Description and factors of variation of the overall health score in French dairy cattle herds using the Welfare Quality ${ }^{\circledR}$ assessment protocol. Prev. Vet. Med., 112, 296-308.

Cook N.B., 2009. Free-stall design for maximum cow comfort. WCDS Advances in Dairy Technology, 21, 255-268.

Cook N.B., Bennett T.B., Nordlund K.V., 2004. Effect of free stall surface on daily activity patterns in dairy cows with relevance to lameness prevalence. J. Dairy Sci., 87, 2912-2922.

Cook N.B., Bennett T.B., Nordlund K.V., 2005. Monitoring indices of cow comfort in free-stall housed dairy herds. J. Dairy Sci., 88, 3876-3885.

Cook N.B., Marin M.J., Mentink R.L., Bennett T.B., Schaefer M.J., 2008. Comfort zonedesign free stalls: do they influence the stall use behavior of lame cows? J. Dairy Sci., 91, 4673-4678.

Dodd F.H., Higgs T.M., Bramley A.J., 1984. Cubicle management and coliform mastitis. Vet. Rec., 114, 522-523.

Drissler M., Gaworski M., Tucker C.B., Weary D.M., 2005. Freestall maintenance: effects on lying behavior of dairy cattle. J. Dairy Sci., 88, 2381-2387.

Espejo L.A., Endres M.I., Salfer J.A., 2006. Prevalence of lameness in high-producing Holstein cows housed in freestall barns in Minnesota. J. Dairy Sci., 89, 3052-3058.

Ferrari P., Moscatelli G., 2009. Gestion du fumier, des conditions d'hygiène et des émissions de gaz à effet de serre dans les exploitations laitières par l'utilisation de fractions solides du lisier recyclées en litière pour vaches. In : Colloque Europ. Bâtiments d'élevage du futur, Lille, France, 133-140.

Fregonesi J.A., Von Keyserlingk M.A.G., Tucker C.B., Veira D.M., Weary D.M., 2009. Neck-rail position in the free stall affects standing behavior and udder and stall cleanliness. J. Dairy Sci., 92, 1974-1985.

Galindo F., Broom D.M., Jackson P.G.G., 2000 A note on possible link between behavior and the occurrence of lameness in dairy cows. Appl. Anim. Behav. Sci., 67, 335-341.
Haley D.B., Rushen J., De Passillé A.M., 2000 Behavioural indicators of cow comfort: activity and resting behaviour of dairy cows in two types of housing. Can. J. Anim. Sci., 80, 257-263.

Haley D.B., De Passillé A.M., Rushen J., 2001. Assessing cow comfort: effects of two floor types and two tie-stall design on the behaviour of lactating dairy cows. Appl. Anim. Behav. Sci., 71, 105-117.

Hulsen J., 2006, Signes de pieds : guide pratique pour des onglons en bonne santé. Roodbont éditions, Zutphen, Pays-Bas, 40p.

Husfeldt A.W., Endres M.I., 2012. Association between stall surface and some animal welfare measurements in freestall dairy herds using recycled manure solids for bedding. J. Dairy Sci., 95, 5626-5634.

Krohn C.C., Munksgaard L., 1993. Behaviour of dairy cows kept in extensive (loose housing pasture) or intensive (tie stall) environments II. Lying and lying-down behaviour. Appl. Anim. Behav. Sci., 37, 1-16.

Kristula M.A., Dou Z., Toth J.D., Smith B.I, Harvey N., Sabo M., 2008. Evaluation of freestall mattress bedding treatments to reduce mastitis bacterial growth. J. Dairy Sci., 91, 1885-1892.

Lensink J., Mounier L., 2012. Les revêtements en caoutchouc dans les logettes et les couloirs de circulation en élevages bovins. Bulletin des GTV, 67, 93-100.

Lidfors L., 1989. The use of getting up and lying down movements in the evaluation of cattle environments. Vet. Res. Com., 13, 307-324.

Manninen E., De Passillé A.M., Rushen J. Norring M., Saloniemi H., 2002. Preferences of dairy cows kept in unheated building for different kind of cubicle flooring. Appl. Anim. Behav. Sci., 75, 281-292.

Martens L., Daelemans J., Maton A., 1980. Les investissements dans les étables pour vaches laitières et leur équipement. Revue de 1'Agriculture, 33, 433-450.

Metcalf J. A., Roberts S.J., Sutton J.D., 1992. Variations in blood flow to and from the bovine mammary gland measured using transit time ultra-sound and dye dilution. Res. Vet. Sci., 53 , $59-63$.

Milklait 2020, 2013. Manuel sur la litière pour les exploitations laitières du NouveauBrunswick, 61p. www.milk2020.ca/fr/dwn/ FRENCHMANUALlr.pdf

Norring M., Manninen E., De Passillé A.M. Rushen J., Saloniemi H., 2008. Effects of sand and straw bedding on the lying behavior, cleanliness, and hoof and hock injuries of dairy cows. J. Dairy Sci., 91, 570-576.

Norring M., Manninen E., De Passillé A.M. Rushen J., Saloniemi H., 2010. Preferences of dairy cows for three stall surface materials with small amounts of bedding. J. Dairy Sci. $93,70-74$

Rodriguez F., 2014. Rest assured: bedding options for robotic milking activities. Progressive Dairyman.

Rulquin H., Caudal J.P., 1992. Effects of lying or standing on mammary blood flow and heart rate of dairy cows. Ann. Zoot., 41, 101.
Rushen J., Haley D., De Passillé A.M., 2007. Effect of softer flooring in tie stalls on resting behavior and leg injuries of lactating cows. J. Dairy Sci., 90, 3647-3651.

Ruud L.E., Boe K.E., Østeras O., 2010. Associations of soft flooring materials in free stalls with milk yield, clinical mastitis, teat lesions, and removal of dairy cows. J. Dairy Sci., 93, 1578-1586.

Scharde S., Zähner M., 2008. Litières dans les logettes pour vaches laitières - compost et fraction solide issue de la séparation du lisier : une alternative. In : Rapport ART, n 699.

Séité Y., Guiocheau S., Gautier B., Foisnon O., Coutant S., Prudhomme J.F., Capdeville J., Ménard J.L., Charlery J., 2012. Concevoir et installer des logettes. Institut de l'élevage (Ed), $12 \mathrm{p}$.

The Dairy Group, 2012. Dairy cow housing Report August 12, 146p. www.dairyco.org.uk non_umbraco/download.aspx?media $=13205$

Tucker C.B., Weary D.M., 2001. Stall design: enhancing cow comfort. Adv. Dairy Tech., $13,155-168$

Tucker C.B., Weary D.M., Fraser D., 2003. Effects of three types of free-stall surfaces on preferences and stall usage by dairy cows. J. Dairy Sci., 86, 521-529.

Tucker C.B., Weary D.M., 2004. Bedding on geotextile mattresses: how much is needed to improve cow comfort? J. Dairy Sci., 87, 28892895.

Tucker C.B., Weary D.M., Fraser D., 2005. Influence of neck-rail placement on free-stall preference, use, and cleanliness. J. Dairy Sci. $88,2730-3737$.

Tucker C.B., Zdnanowicz G., Weary D.M., 2006. Brisket boards reduce freestall use. J. Dairy Sci., 89, 2603-2607.

Tucker C.B., Weary D.M., Von Keyserlingk M.A.G., Beauchemin K.A., 2009. Cow comfort in tie-stall: increased depth of shavings or straw bedding increases lying time. J. Dairy Sci., 92, 2684-2690.

Veissier I., Capdeville J., Delval E., 2004. Cubicle housing system for cattle: comfort of dairy cows depends on cubicle adjustment. J. Anim. Sci., 82, 3321-3337.

Veissier I., Botreau R., Perny P., 2010. Evaluation multicritère appliquée au bien-être des animaux en ferme ou à l'abattoir : difficultés et solutions du projet Welfare Quality ${ }^{\circledR}$. INRA Prod. Anim., 23, 269-284

Vin H., Vin-Dekoker J., 2007. Logement et infection mammaire. In : JNGTV, Nantes, France, 245-253

Weary D.M., Taszkun I., 2000. Hock lesions and free-stall design. J. Dairy Sci., 83, $697-$ 702 .

Zdanowicz M., Shelford J.A., Tucker C.B., Weary D.M., Von Keyserlingk M.A.G., 2004. Bacterial populations on teat ends of dairy cows housed in free stalls and bedded with either sand or sawdust. J. Dairy Sci., 87, 16941701. 


\title{
Résumé
}

Réalisant un bon compromis entre les besoins des animaux et ceux des éleveurs, la stabulation libre à logettes s'est imposée comme une des principales modalités d'hébergement des vaches laitières. Les recommandations relatives à sa mise en place et à sa gestion font cependant toujours l'objet de révisions. Une attention particulière est actuellement accordée à l'amélioration des conditions de confort de la vache. Le confort de la vache couchée peut être évalué par le temps passé en position couchée, la fréquence des changements de position et la facilité avec laquelle les vaches se couchent et se lèvent. Le confort de couchage est principalement lié à la souplesse du sol, aux dimensions des logettes et à la façon de les délimiter tant latéralement qu'à l'avant. Un sol suffisamment souple peut être assuré soit par des matelas multicouches, soit par des litières épaisses. Les matelas doivent être recouverts d'un matériau de litière absorbant l'humidité en surface et contribuant à garantir la propreté du sol et de la mamelle. Pour atteindre ces objectifs, la nature du matériau (paille, sciure, sable, chaux...) apparaît moins importante que la fréquence de renouvellement. Les logettes " creuses ", dont le sol est recouvert d'une couche épaisse $( \pm 20 \mathrm{~cm})$ de litière, semblent globalement mieux appréciées par les vaches que celles équipées de matelas et leur utilisation est associée à une moindre fréquence de lésions au niveau des membres ainsi que de boiteries. Le sable apparaît comme un matériau de premier choix pour assurer le confort des animaux. Néanmoins, l'entretien des logettes creuses demande un surcroît de travail et la manutention de sable comme matériau de litière nécessite des équipements spécifiques. Les recommandations relatives aux dimensions des logettes entrent dans une fourchette de valeurs assez étendue car devant être adaptées au gabarit des animaux et intégrer la recherche de conditions optimisant la propreté du bâti. Les modèles de séparations latérales sont à évaluer quant à leur efficacité à contraindre l'animal à ne pas déborder sur les emplacements voisins tout en évitant qu'il ne se blesse ou ne soit gêné lors du lever et du coucher. Enfin, le dispositif limitant l'avancement dans la logette sera jugé sur son efficacité à dégager l'espace nécessaire pour permettre à l'animal de projeter sans contrainte la tête vers l'avant lors du relevé.

\begin{abstract}
Cubicle design and the comfort of dairy cows

Cubicle housing offers a good compromise between the needs of the animals and those of the breeders; it is one of the main housing systems for dairy cows. The recommendations relative to the design and management of cubicle housing are, however, always subject to revision. Special attention is now being given to the improvement of animal comfort, particularly with regards to the lying cow. In this context, the level of comfort is estimated by the duration of the lying time, the frequency of the lying bouts and the ease with which cows are able to get up and lie down. The level of cow comfort is in direct relation to the softness of the floor, to the cubicle dimensions, and to the design of the lateral and frontal partitions. A soft floor can be assured through the use of either multilayer mattresses or a thick litter. Mattresses must be covered with a litter material that absorbs surface moisture and helps to ensure the cleanliness of the stall and udder. To achieve these objectives, the nature of the litter material (straw, sawdust, sand, lime, etc.) appears to be less important than the frequency of renewal. Cubicle floors covered with a thick layer $( \pm 20 \mathrm{~cm})$ of litter seem generally better appreciated by the cows than those equipped with mattresses, and their use is associated with a lower frequency of leg injuries as well as lameness. Sand appears to be a top-grade material for ensuring animal comfort. Nevertheless, the maintenance of a thick layer of litter demands an extra daily labor requirement and the handling of sand as a litter material requires specific equipment. There is a wide range of recommendations on cubicle dimensions due to differences in cow size and the need to take into account the cleanliness of the floor. Lateral partitions must be evaluated for their effectiveness in constraining the animal from spilling out into the neighboring cubicles while at the same time avoiding both injury and difficulties experienced by the cow in getting up and lying down. Finally, frontal partitions must be evaluated regarding their effectiveness in providing sufficient space to allow the cow to thrust its head forward as it stands up.
\end{abstract}

CAZIN P., NICKS B., DUFRASNE I., 2014. Aménagement des logettes et confort des vaches laitières. INRA Prod. Anim., 27, 359-368. 